\section{ARTÍCULOE ESPEGIAL}

1. Médico Gineco-Obstetra, Hospita Nacional Daniel Alcides Carrión-Callao. Centro de Medicina Fetal: CENMEF. Clínica Santa Isabel, Lima, Perú. ORCID: 0000-0003-3321-3355

2. Director de la Unidad de ultrasonido OBGYN, Hospital Maternidad Lis. Tel Aviv Medical Center, Israel. ORCID: 00000003-0864-206X

Fuente de financiamiento: Autofinanciado

Conflicto de interés: Ninguno

Recibido: 20 junio 2021

Aceptado: 6 agosto 2021

Publicación en línea:

Correspondencia:

Rommel Lacunza Paredes

- Av. Guardia Chalaca 2176, Bellavista. Callao

용 985436784

\rrlacunza@hotmail.com

Citar como: Lacunza Paredes R, Malinger G. Evaluación del cerebrofetal: nuevas herramientas. Rev Peru Ginecol Obstet. 2021;67(4). DOI: https://doi.org/10.31403/rpgo.v67i2370

\section{Evaluación del cerebro fetal: nuevas herramientas Fetal brain assessment: new tools}

Rommel Lacunza Paredes ${ }^{1}$, Gustavo Malinger ${ }^{2}$

DOI: https://doi.org/10.31403/rpgo.v67i2370

\section{RESUMEN}

La evaluación del cerebro fetal es un punto imprescindible en el ultrasonido obstétrico, por la gran cantidad de malformaciones que pueden ser diagnosticadas. La guía de ISUOG nos brinda los cortes elementales para la sospecha de la patología cerebral; pero, podemos ampliar y mejorar nuestro ultrasonido con la visualización de estructuras fácilmente reproducibles, tales como el complejo anterior, cuerpo calloso, cisura de Silvio y el cuarto ventrículo. Presentamos algunas herramientas para complementar la evaluación del cerebro fetal.

Palabras clave. Feto, Desarrollo embrionario y fetal, Cerebro, Cuerpo calloso, Acueducto cerebral, Cuarto ventrículo.

\section{ABSTRACT}

The evaluation of the fetal brain is an essential point in obstetric ultrasound due to the large number of malformations that can be diagnosed. The ISUOG guide provides us with the elementary sections for the suspicion of brain pathology; but we can extend and improve our ultrasound with the visualization of easily reproducible structures, such as the anterior complex, corpus callosum, Sylvian fissure and the fourth ventricle. We present some tools to complement the assessment of the fetal brain.

Key words: Fetus, Embryonic and fetal development, Cerebrum, Corpus callosum, Cerebral aqueduct, Fourth ventricle.

\section{INTRODUCCIÓN}

Las patologías del cerebro fetal son frecuentes (1-2/1 000 nacidos vivos) y requieren en todos los casos una evaluación exhaustiva, debido no solo a las implicancias sino también a su repercusión a largo plazo. Actualmente, la gran mayoría de malformaciones cerebrales diagnosticadas durante el embarazo representan cuadros aparatosos y evidentes. Sin embargo, muchas malformaciones 'sutiles' o 'menores' -las cuales pueden llegar a tener graves repercusiones en el neurodesarrollo a largo plazo afectando la integración en la sociedad de estos niños- no son diagnosticadas durante la evaluación ultrasonográfica de rutina.

En la actualidad, la guía de evaluación rutinaria del cerebro fetal en el segundo trimestre ${ }^{(1)}$, así como la guía para evaluación básica del cerebro fetal (2), propuestas por ISUOG (International Society of Ultrasound in Obstetrics and Gynecology), se limitan a un estudio transabdominal de cortes axiales del cerebro. Pero, como revisaremos a continuación, los alcances de esta evaluación tienen importantes limitaciones y generalmente deben ser ampliados cuando nos enfrentamos a una sospecha de patología cerebral fetal.

La presente revisión no pretende ser un manual de neurosonografía del cerebro fetal (examen amplio y dedicado), la cual es realizada por un experto con experiencia en la patología cerebral(2), sino que tiene como objetivo proporcionar al operador, durante el cribado básico, los fundamentos para establecer una sospecha diagnóstica inicial y de esta forma poder referir a la paciente a un examen especializado. Sugeriremos y sustentaremos algunas herramientas que complementan, aunque no sustituyen, lo recomendado por ISUOG. 


\section{Metodolocía de LA BÚSQUEDA dE INFORMACIÓN}

Se realizó la búsqueda de artículos originales y revisiones en las bases de datos OVIDSP, ScienceDirect, SciELO y PUBMED, con los términos de búsqueda 'neurosonografia fetal', 'complejo anterior', 'cuerpo calloso', 'cisura de Silvio', 'cuarto ventrículo'. Se seleccionó los más relevantes en el ámbito prenatal y perinatal de los últimos 5 años; se incluyeron algunos de más de 10 años por su relevancia histórica y científica.

\section{EvaluACIÓN DEL CEREBRo FETAL}

La evaluación rutinaria del cerebro fetal comienza por la identificación de la línea media, forma e integridad del cráneo. Parte primordial de este primer estudio es la identificación de estructuras cerebrales tales como los tálamos y el cavum de septum pellucidum (CSP). Se realizan las biometrías cefálicas -diámetro biparietal y circunferencia cefálica-, siempre valoradas en percentiles según la edad gestacional (plano transtalámico o biparietal) (figuras 1A y $2 \mathrm{~A}$ ).
La segunda imagen cerebral requiere la identificación del sistema ventricular, en especial del atrio ventricular. Se establece un plano en el cual también se visualiza el CSP y las astas anteriores del sistema ventricular (plano transventricular). Se realiza con la intención de descartar dilataciones del sistema ventricular cerebral (ventriculomegalia, signo muy frecuente y común a muchas patologías cerebrales) ${ }^{(3-10)}$. Los reparos anatómicos para su correcta medida involucran la identificación de la cisura parieto-occipital, que marca el lugar donde se realiza la medida in to in de las paredes del atrio ventricular ${ }^{(1,2)}$; se sigue manteniendo el valor clásico de 9,9 mm como dogma de normalidad( ${ }^{(3-5)}($ figuras $1 \mathrm{~B}$ y $2 \mathrm{~B}$ ). Durante el primer trimestre y hasta la semana 20, este valor no debe ser tenido en cuenta. La sospecha diagnóstica se basa en la apreciación cualitativa del ventrículo lateral y su relación con el plexo coroideo, que normalmente debe ocupar todo el ancho del atrio(11,12).

El tercer plano se enfoca en la fosa posterior: se identifica el cerebelo y se diferencia el vermis de

Figura 1. Cortes recomendados por isuog. A: corte transtalámico. B: corte transventricular. C: corte transcerebeloso.

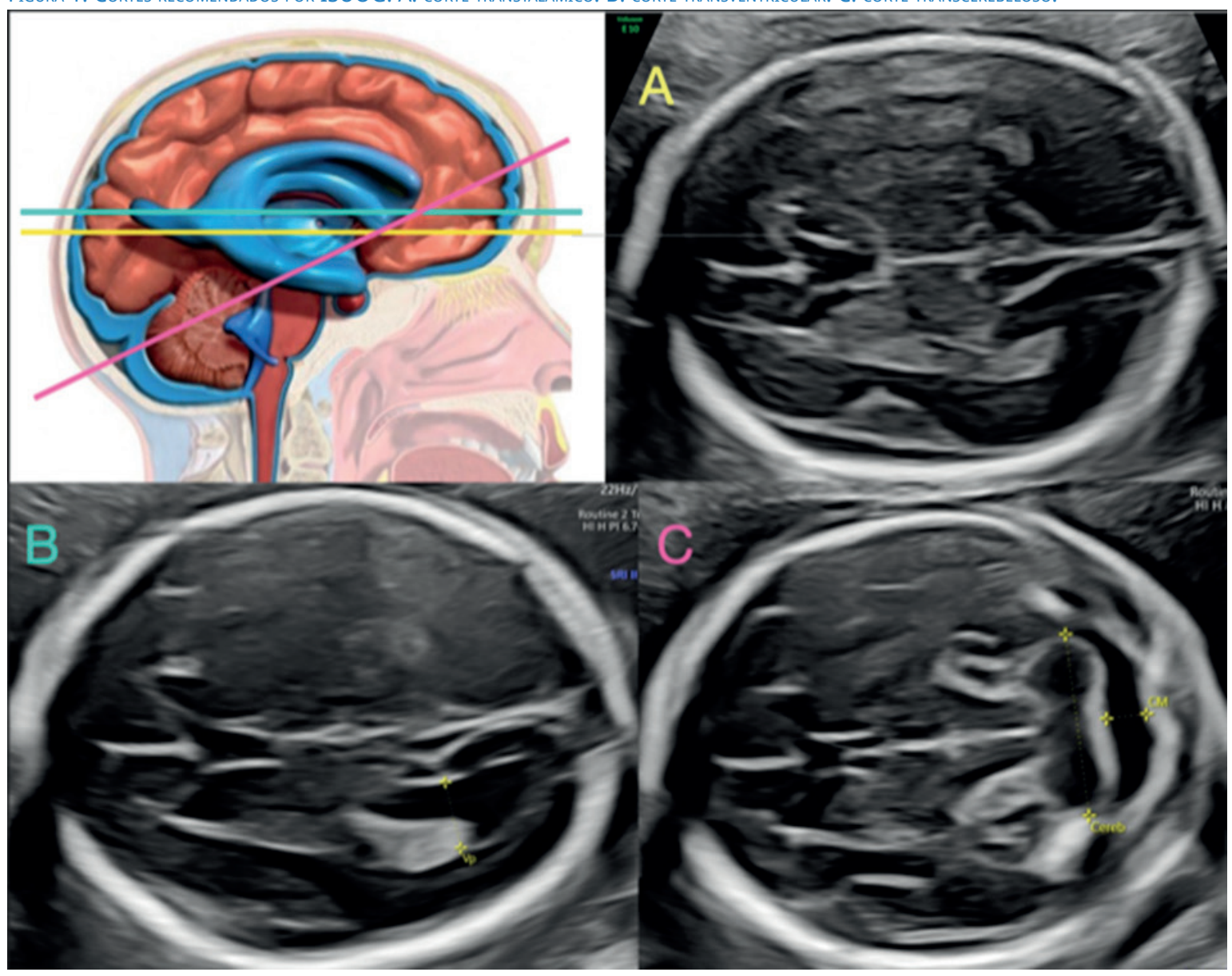

2 Rev Peru Ginecol Obstet. 2021;67(4) 
los hemisferios cerebelosos (estructura central hiperecogénica). Nuevamente, se exige un plano en el cual también se visualiza el CSP y las astas anteriores del sistema ventricular ${ }^{(1,2)}$. Se mide la cisterna magna (CM), que va desde el vermis hasta la tabla interna del hueso occipital (con valor de 2 a $10 \mathrm{~mm}$ en el segundo y tercer trimestre). Tanto el colapso como la dilatación son signos comunes a muchas patologías de la fosa posterior y la columna ${ }^{(13,14)}$. Así mismo, se mide el diámetro transcebeloso (valorando su tamaño en percentiles en relación a la edad gestacional), que incluye los hemisferios y vermis. De forma subjetiva se adiciona la normalidad del cerebelo valorando la forma de su contorno y la no 'comunicación' del cuarto ventrículo con la cisterna magna $^{(15)}$ (figuras $1 \mathrm{C}$ y $2 \mathrm{C}$ ).

\section{Evaluación del 'COMPLejo ANTERIOR'}

Durante la evaluación del cerebro, la identificación del CSP es un punto de reparo cardinal para los tres cortes recomendados por ISUOG ${ }^{(1,2)}$; sin em- bargo, prestamos poca atención a la información que esta estructura nos ofrece. Debemos empezar a valorar la relación de los cuernos anteriores del sistema ventricular y el CSP, lo que Cagneaux y Guibaud ${ }^{(16)}$ denominaron 'complejo anterior'. En este complejo anterior existen estructuras clave que debemos identificar, como la rodilla del cuerpo calloso, la integridad de la línea media y la forma de los cuernos anteriores (figura 3). Viñals ${ }^{(17)}$ describe la morfología normal del complejo anterior, dejando en claro que el CSP puede ser de forma rectangular o de un triángulo de base anterior, y las astas anteriores del sistema ventricular parecen una 'coma' o un triangulo (figuras 3B y $3 \mathrm{C}$ ).

Esta evaluación cualitativa rápida aporta importante información sobre la existencia de al menos una porción del cuerpo calloso, y la simetría del sistema ventricular y de la zona periventricular, que puede ser asiento de patología del desarrollo cortical, hemorrágica o infecciosa ${ }^{(18,19)}$. Todo esto lo posiciona como una herramienta con potencial para la detección de

Figura 2. Análisis de imagen volumétrica del cerebro fetal, donde se muestra el nivel de CAda corte recomendado por ISUOG. A: CoRTe transTALÁmico. B: CORTE TRANSVENTRICULAR. C: CORTE TRANSCEREBELOSO.

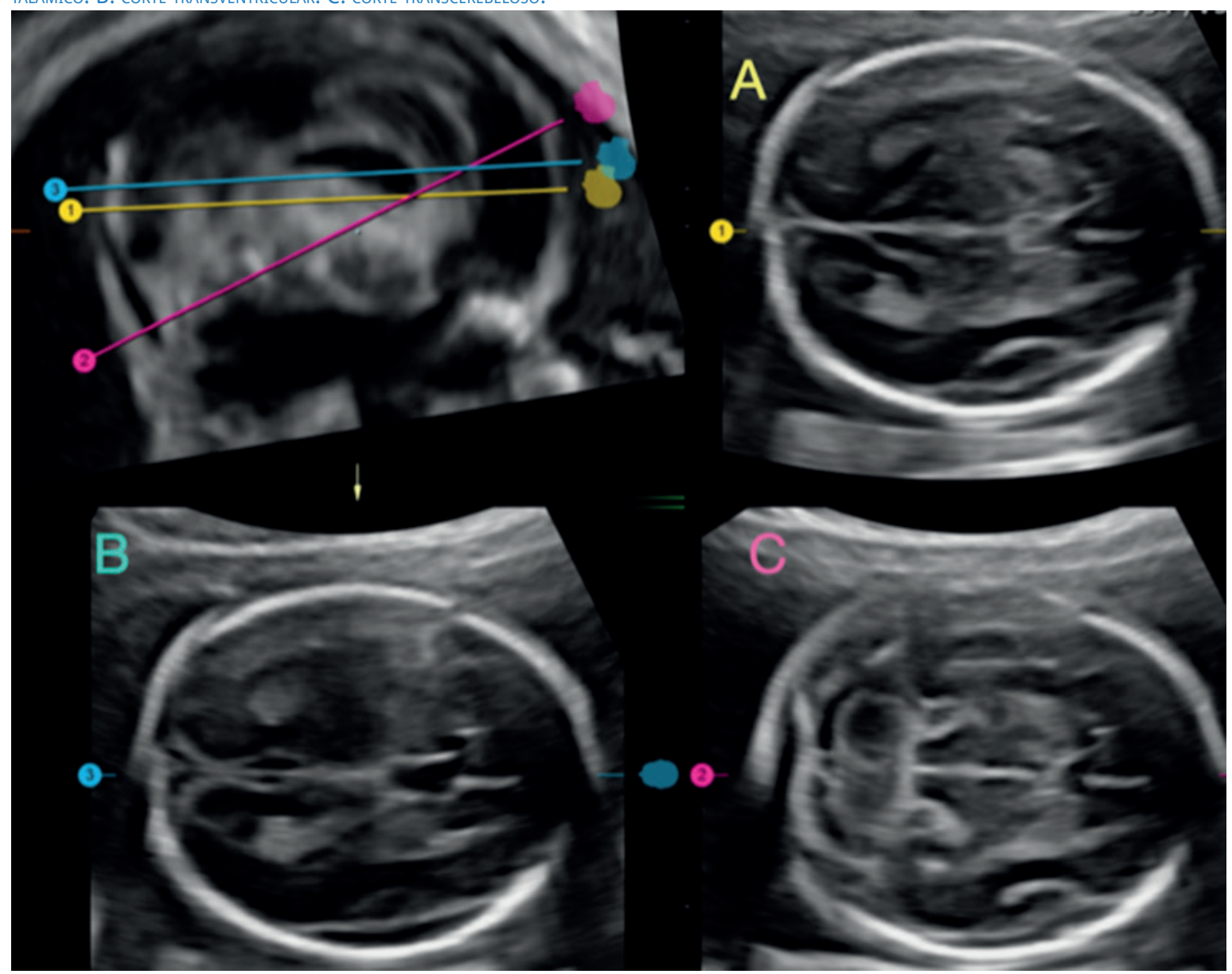

Rev Peru Ginecol Obstet. 2021;67(4) 3 
Figura 3. Complejo anterior. A: Corte transventricular donde se visualiza el complejo anterior. B y C: Estructuras que Se identifican en el

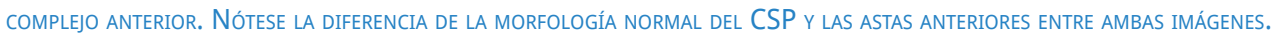

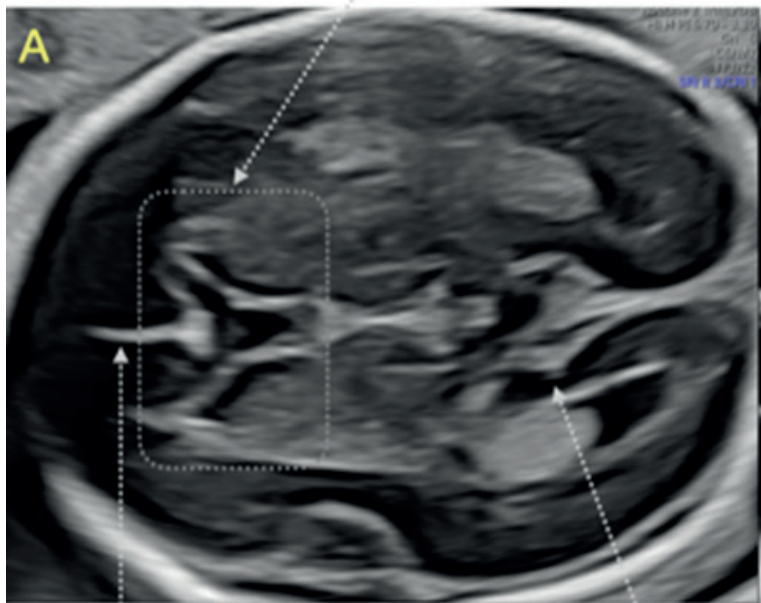

Cisura parieto occipital

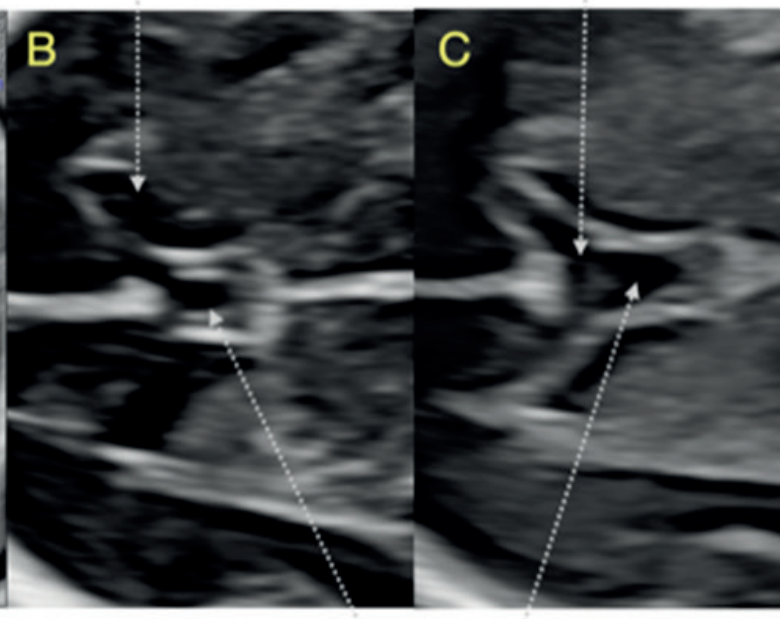

Cavum septi pellucidi holoprosencefalia(20,21), anomalías comisurales (agenesia completa del cuerpo calloso, displasia septo-preóptica)(22-28), esquisencefalia( ${ }^{(29)}$ y barotrauma (ventriculomegalia obstructiva) ${ }^{(30,31)}$.

\section{Visualización del CUERPo CALLOSO}

Dentro de la evaluación de la cara fetal recomendada por ISUOG(1), el perfil fetal es una visión obligada, constituyendo una oportunidad valiosa para la visualización del cuerpo calloso (CC) (figu- ra 4A). Si estamos en un estricto corte sagital y logramos aprovechar la sutura metópica o la fontanela anterior, la visualización del CC será generalmente adecuada, y de forma rápida podremos constatar su presencia, morfología normal y relación con estructuras cercanas, estableciendo sospecha de agenesia parcial, total o disgenesia(32-38). Así también, como recomiendan Youssef y Pilu(39), podría adicionarse el uso del Doppler para facilitar la visualización por la forma de la vasculatura (arterias pericallosas) (figura 4B).

Figura 4. A: Corte sagital del cerebro fetal donde se logra la visualización del cuerpo calloso en un corte de perfil fetal. B: Visualización de LA ARTERIA PERICALLOSA.

Rodilla del cuerpo calloso Arteria pericallosa

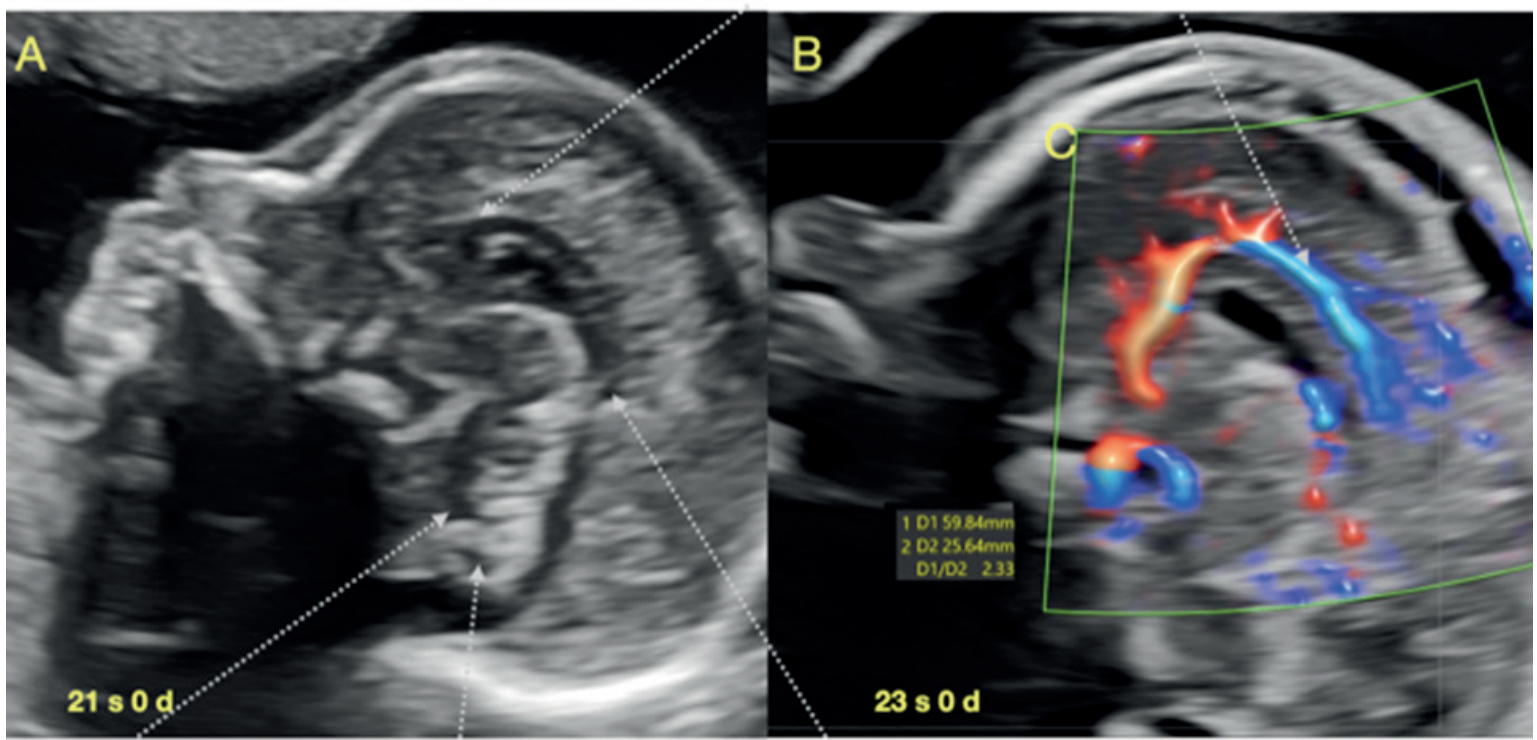

Fastigium

Vermis cerebeloso

Esplenio del cuerpo calloso

4 Rev Peru Ginecol Obstet. 2021;67(4) 
Si quisiéramos aplicar alguna medida sencilla más allá de la visualización directa de la estructura, en relación a las dimensiones del CC, nos permitimos sugerir valorar la relación entre la longitud de cuerpo calloso con el diámetro anteroposterior, en un corte sagital. Esta relación se mantiene constante entre 3,4 y 3,8 desde la semana 20 hasta el término y guarda relación con los valores encontrados en neonatos ${ }^{(40-42)}$.

\section{Visualización de la cisura de Silvio}

La evaluación de la corteza cerebral siempre ha sido un tópico complejo y de sistematización esquiva ${ }^{(43-50)}$. Al momento de realizar la evaluación del atrio ventricular, visualizamos necesariamente la cisura parieto-occipital como un punto de referencia y tenemos una idea intuitiva de la correspondencia de su forma con la edad gestacional(43-45). Podemos adicionar a esto la visualización de la cisura de Silvio (CS) en una visión axial fácilmente reproducible ${ }^{(51,52)}$.
Quarello(53), en el año 2008 valoró en 200 fetos la evolución de la relación entre la ínsula y el lóbulo temporal (operculización de la CS), desde las 22 a 32 semanas, y nos propone un puntaje de 0 a 10 relacionado a la edad gestacional. Esta evaluación se da en un corte axial, un poco más caudal a la vista transtalámica, en el cual debemos lograr visualizar tres puntos de estandarización del nivel de la CS: las columnas del fórnix en la parte inferior del CSP, tercer ventrículo en la parte central y la cisterna ambiens en la parte posterior (figura 5). Guibaud (54) simplifica la tabla de valoración de la CS (figura 5A) y la aplica contra diversas malformaciones cerebrales, mostrando que el anormal desarrollo de la operculización de la CS se encuentra no solo en malformaciones corticales sino también en patología comisural, defectos del tubo neural y de la fosa posterior ${ }^{(47,49,52,55)}$. Por ello, la evolución de la operculización de la CS es un elemento valioso, reproducible y efectivo en la sospecha de patología cerebral fetal.

Figura 5. ImÁgenes representativas del cambio de morfología de la cisura de Silvio conforme progresa La edad GeStaCional. A: Esquema proPUESTO POR GUibaud ${ }^{(54)}$, PARA la VAloración de la Cisura de Silvio.
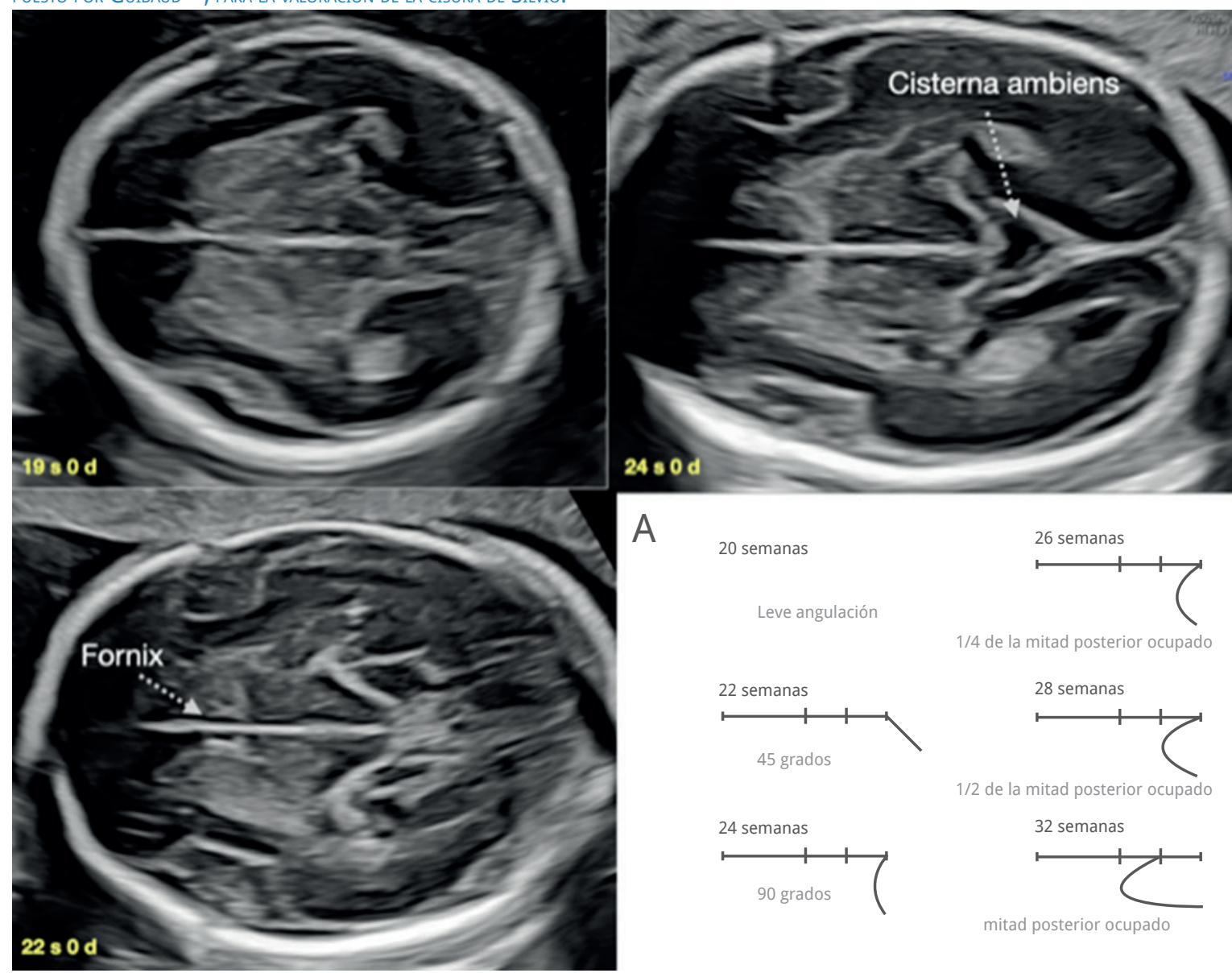
Figura 6. A: VisuALIZACIÓN DEL CUARTO VENTRÍCULO Y COMO REALIZAR LA MEDICIÓN DE LOS DIÁMETROS. B: LÍNEA ROSA REPRESENTA EL NIVEL DEL CORTE

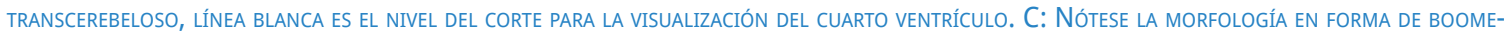
RANG DEL CUARTO VENTRÍCULO

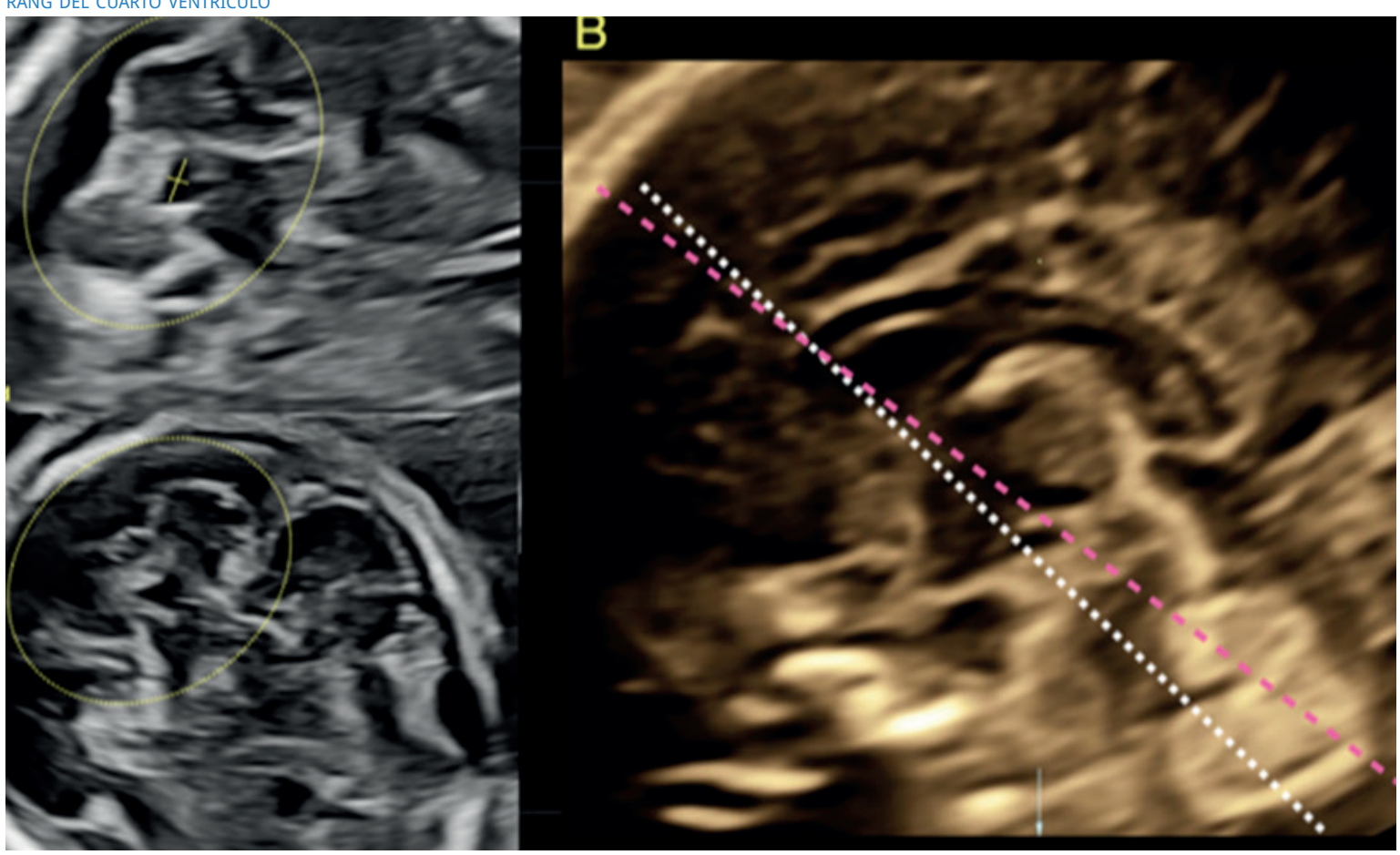

\section{Evaluación del cuarto ventrículo cere- BRAL FETAL}

La evaluación de la fosa posterior presenta limitaciones, especialmente en los casos que tienen medidas de CM menores a $10 \mathrm{~mm}$, dado que se tiende a evaluar únicamente la presencia del vermis superior, dejando de lado las alteraciones en posición (rotación) y forma ${ }^{(56-58)}$. La valoración de la forma del cuarto ventrículo en una visión axial puede darnos información adicional, al ser un indicador indirecto de la normalidad en el vermis y mesencéfalo (pedúnculos cerebrales), aunque la valoración sagital es lo ideal para una evaluación avanzada(59-61).

En 1994, Baumeister ${ }^{(62)}$ describió la técnica para la evaluación del cuarto ventrículo en un corte axial, por medio de la inclinación del corte del diámetro transcerebeloso hasta lograr la visualización del cuarto ventrículo y los hemisferios cerebelosos (figuras $6 \mathrm{~A}$ y $6 \mathrm{C}$ ). Se identifica 3 formas: ovoide, triangular y boomerang (figuras 6A y 6B). Quarello(63) resalta la forma normal cuadrangular del cuarto ventrículo con el diámetro transverso de mayor tamaño que el antero-posterior y, en los casos con síndrome de Joubert, observó que la forma del cuarto ventrículo era alargada. Haratz ${ }^{(64)}$, en base a estos estudios, en una serie prospectiva de $384 \mathrm{fe}$ tos normales propone la valoración rápida del cuarto ventrículo por la relación entre diámetro latero-lateral y antero-posterior, denominándolo indice del cuarto ventrículo (fourth ventricle índex - 4VI), el cual es mayor de 1 en fetos normales, independiente de la edad gestacional (figura 6A). Por medio de esa evaluación se podría lograr la detección de patologías como el síndrome de Joubert, romboencefalosinapsis, hipoplasia pontocerebelar y malformaciones corticales de tipo adoquinado ${ }^{(65-70)}$.

\section{ConCLusión}

En la evaluación del cerebro fetal durante el tamizaje con ultrasonido, es factible examinar el complejo anterior, cuerpo calloso, cisura de Silvio y cuarto ventrículo (no es obligada su evaluación de acuerdo a las guías ISUOG). Ello potencialmente tiene impacto en la sospecha diagnóstica de algunas de las principales anomalías neurológicas fetales. Estas visualizaciones son complementarias a las recomendadas por ISUOG y, al ser generalmente reproducibles en la práctica clínica diaria, pueden ampliar nuestra capacidad de detección de patología cerebral fetal. 


\section{ReferenCiAs BIBLIOGRÁfiCAS}

1. Salomon LJ, Alfirevic Z, Berghella V, Bilardo C, Hernandez-Andrade E, Johnsen SL, et al. Practice guidelines for performance of the routine mid-trimester fetal ultrasound scan. Ultrasound Obstet Gynecol. 2011;37(1):116-26. doi:10.1002/uog.8831

2. Malinger G, Paladini D, Haratz KK, Monteagudo A, Pilu G, Timor-TritschIE. ISUOG Practice Guidelines (updated): sonographic examination of the fetal central nervous system. Part 1: performance of screening examination and indications for targeted neurosonography. Ultrasound Obstet Gynecol. 2020;56:476-84. Doi:10.1002/uog.22145

3. Pisapia JM, Sinha S, Zarnow DM, Johnson MP, Heuer GG. Fetal ventriculomegaly: Diagnosis, treatment, and future directions. Childs Nerv Syst. 2017;33(7):1113-23. doi:10.1007/ s00381-017-3441-y

4. Guibaud L, Lacalm A, Rault E. Fetal ventriculomegaly: diagnostic, ethical and semantic considerations. Childs Nerv Syst. 2017;33(11):1863-4. doi:10.1007/s00381-017-3500-4

5. Malinger G, Lerman-Sagie T. Fetal neurology. Eur J Paediatr Neurol. 2018;22(6):895-7. doi:10.1016/j.ejpn.2018.11.004

6. D'Antonio F, Zafeiriou DI. Fetal ventriculomegaly: What we have and what is still missing. Eur J Paediatr Neurol. 2018;22(6):898-9. doi:10.1016/j.ejpn.2018.11.005

7. Griffiths PD, Bradburn M, Campbell MJ, Cooper Cl, Graham $R$, Jarvis $D$, et al. Use of MRI in the diagnosis of fetal brain abnormalities in utero (MERIDIAN): a multicentre, prospective cohort study. Lancet.2017;389(10068):538-46. doi:10.1016/S0140-6736(16)31723-8

8. Paladini D, Malinger G, Pilu G, Timor-Trisch I, Volpe P. The MERIDIAN trial: caution is needed. Lancet. 2017;389(10084):2103. doi:10.1016/S0140-6736(17)31337-5

9. Carta S, Kaelin Agten A, Belcaro C, Bhide A. Outcome of fetuses with prenatal diagnosis of isolated severe bilateral ventriculomegaly: systematic review and meta-analysis Ultrasound Obstet Gynecol. 2018;52(2):165-73. doi:10.1002/ uog. 19038

10. Scala C, Familiari A, Pinas A, Papageordhiou AT, Bhide A, TGhilaganathan B, Khalil A. Perinatal and long-term outcomes in fetuses diagnosed with isolated unilateral ventriculomegaly: systematic review and meta-analysis. Ultrasound Obstet Gynecol. 2017;49(4):450-9. doi:10.1002/uog.15943

11. Manegold-Brauer G, Oseledchyk A, Floeck A, Berg C, Gembruch U, Geipel A. Approach to the sonographic evaluation of fetal ventriculomegaly at 11 to 14 weeks gestation. BMC Pregnancy Childbirth. 2016;16(12):3. doi:10.1186/s12884016-0797-z

12. Chaoui R, Benoit B, Entezami M, Frenzel W, Heling KS, Ladendorf $B$, et al. Ratio of fetal choroid plexus to head size: simple sonographic marker of open spina bifida at 11-13 weeks gestation. Ultrasound Obstet Gynecol. 2020;55(1):816. doi:10.1002/uog.20856

13. Wüest A, Surbek D, Wiest R, Weisstanner C, Bonel H, Steinlin M, Raio L, Tutschek B. Enlarged posterior fossa on prenatal imaging: differential diagnosis, associated anomalies and postnatal outcome. Acta Obstet Gynecol Scand. 2017;96(7):837-43. doi:10.1111/aogs.13131
14. Garel C. Posterior fossa malformations: main features and limits in prenatal diagnosis. Pediatr Radiol. 2010;40(6):103845. doi:10.1007/s00247-010-1617-7

15. Leibovitz Z, Guibaud L, Garel C, Massoud M, Karl K, Malinger $\mathrm{G}$, et al. The cerebellar "tilted telephone receiver sign" enables prenatal diagnosis of PHACES syndrome. Eur J Paediatr Neurol. 2018;22(6):900-9. doi:10.1016/j.ejpn.2018.08.006

16. Cagneaux M, Guibaud L. From cavum septi pellucidi to anterior complex: how to improve detection of midline cerebral abnormalities. Ultrasound Obstet Gynecol. 2013;42(4):485-6. doi:10.1002/uog.12505

17. Viñals F, Correa F, Gonçalves-Pereira PM. Anterior and posterior complexes: a step towards improving neurosonographic screening of midline and cortical anomalies. Ultrasound Obstet Gynecol. 2015;46(5):585-94. doi:10.1002/ uog. 14735

18. Levy M, Lev D, Leibovitz Z, Kashanian A, Gindes L, Tamarkin $M$, et al. Periventricular pseudocysts of noninfectious origin: Prenatal associated findings and prognostic factors. Prenat Diagn. 2020;40(8):931-41. doi:10.1002/pd.5704

19. Khalil A, Sotiriadis A, Chaoui R, da Silva Costa F, D'Antonio F, Heath PT, et al. ISUOG Practice Guidelines: role of ultrasound in congenital infection. Ultrasound Obstet Gynecol. 2020;56(1):128-51. doi:10.1002/uog.21991

20. Winter TC, Kennedy AM, Woodward PJ. Holoprosencephaly: a survey of the entity, with embryology and fetal imaging. Radiographics. 2015;35(1):275-90. doi:10.1148/ rg.351140040

21. Malinger G, Lev D, Kidron D, Heredia F, Hershkovitz R, Lerman-Sagie T. Differential diagnosis in fetuses with absent septum pellucidum. Ultrasound Obstet Gynecol. 2005;25(1):42-9. doi:10.1002/uog.1787

22. Malinger G, Lev D, Oren M, Lerman-Sagie T. Non-visualization of the cavum septi pellucidi is not synonymous with agenesis of the corpus callosum. Ultrasound Obstet Gynecol. 2012;40(2):165-70. doi:10.1002/uog.11206

23. Borkowski-Tillman T, Garcia-Rodriguez R, Viñals F, Branco $M$, Kradjen-Haratz K, Ben-Sira L, et al. Agenesis of the septum pellucidum: Prenatal diagnosis and outcome. Prenat Diagn. 2020;40(6):674-80. doi:10.1002/pd.5663

24. Ben M'Barek I, Tassin M, Guët A, Simon I, Mandelbrot L, Ponce O. Antenatal diagnosis of absence of septum pellucidum. Clin Case Rep. 2020;8(3):498-503. doi:10.1002/ccr3.2666

25. Sundarakumar DK, Farley SA, Smith CM, Maravilla KR, Dighe MK, Nixon JN. Absent cavum septum pellucidum: a review with emphasis on associated commissural abnormalities. Pediatr Radiol. 2015;45(7):950-64. doi:10.1007/s00247-0153318-8

26. Maduram A, Farid N, Rakow-Penner R, Ghassemi N, Khanna PC, Robbins SL, Hull A, Gold J, Pretorius DH. Fetal ultrasound and magnetic resonance imaging findings in suspected septo-optic dysplasia: a diagnostic dilemma. J Ultrasound Med. 2020;39(8):1601-14. doi:10.1002/jum.15252

27. Pilliod RA, Pettersson DR, Gibson T, Gievers L, Kim A, Schaey $\mathrm{R}$, Oh KY, Shaffer BI. Diagnostic accuracy and clinical outcomes associated with prenatal diagnosis of fetal absent cavum septi pellucidi. Prenat Diagn. 2018;38(6):395-401. doi:10.1002/pd.5247 
28. Paladini D, Birnbaum R, Donarini G, Maffeo I, Fulcheri E. Assessment of fetal optic chiasm: an echoanatomic and reproducibility study. Ultrasound Obstet Gynecol. 2016;48(6):72732. doi:10.1002/uog.17227

29. Braga VL, da Costa MDS, Riera R, Dos Santos Rocha LP, de Oliveira Santos BF, Matsumura Hondo TT, et al. Schizencephaly: A review of 734 patients. Pediatr Neurol. 2018;87:23-9. doi:10.1016/j.pediatrneurol.2018.08.001

30. Rault E, Lacalm A, Massoud M, Massardier J, Di Rocco F, Gaucherand P, Guibaud L. The many faces of prenatal imaging diagnosis of primitive aqueduct obstruction. Eur J Paediatr Neurol. 2018;22(6):910-8. doi:10.1016/j.ejpn.2018.07.015

31. Kline-Fath BM, Arroyo MS, Calvo-Garcia MA, Horn PS, Thomas C. Congenital aqueduct stenosis: Progressive brain findings in utero to birth in the presence of severe hydrocephalus. Prenat Diagn. 2018;38(9):706-12. doi:10.1002/pd.5317

32. Malinger G, Lev D, Lerman-Sagie T. The fetal corpus callosum. 'The truth is out there'. Ultrasound Obstet Gynecol. 2007;30(2):140-41. doi:10.1002/uog.4095

33. Lerman-Sagie T, Ben-Sira L, Achiron R, L Schreiber, G Hermann, D Lev, et al. Thick fetal corpus callosum: an ominous sign? Ultrasound Obstet Gynecol. 2009;34(1):55-61. doi:10.1002/uog.6356

34. Kidron D, Shapira D, Ben Sira L, Malinger G, Lev D, Cioca A, Sharony R, Lerman Sagie T. Agenesis of the corpus callosum. An autopsy study in fetuses. Virchows Arch. 2016;468(2):219-30. doi:10.1007/s00428-015-1872-y

35. Shinar S, Har-Toov J, Lerman-Sagie T, Malinger G. Thick corpus callosum in the second trimester can be transient and is of uncertain significance. Ultrasound Obstet Gynecol. 2016;48(4):452-7. doi:10.1002/uog.15678

36. Masmejan S, Blaser S, Keunen J, Seaward G, Windrim R, Kelly E, Ryan G, Baud D, Van Mieghem T. Natural history of ventriculomegaly in fetal agenesis of the corpus callosum. J Ultrasound Med. 2020;39(3):483-8. doi:10.1002/jum.15124

37. Karl K, Esser T, Heling KS, Chaoui R. Cavum septi pellucidi (CSP) ratio: a marker for partial agenesis of the fetal corpus callosum. Ultrasound Obstet Gynecol. 2017;50(3):336-41. doi:10.1002/uog.17409

38. Schupper A, Konen O, Halevy A, Cohen R, Aharoni S, Shuper A. Thick corpus callosum in children. J Clin Neurol. 2017;13(2):170-4. doi:10.3988/jen.2017.13.2.170

39. Youssef A, Ghi T, Pilu G. How to image the fetal corpus callosum. Ultrasound Obstet Gynecol. 2013;42(6):718-20. doi:10.1002/uog.12367

40. Malinger G, Zakut $\mathrm{H}$. The corpus callosum: normal fetal development as shown by transvaginal sonography. AJR Am J Roentgenol. 1993;161(5):1041-3. doi:10.2214/ ajr.161.5.8273605

41. Tepper R, Leibovitz Z, Garel C, Sukenik-Halevy R. A new method for evaluating short fetal corpus callosum. Prenat Diagn. 2019;39(13):1283-90. doi:10.1002/pd.5598

42. Gao Y, Yan K, Yang L, Cheng G, Zhou W. Biometry reference range of the corpus callosum in neonates: An observational study. Medicine (Baltimore). 2018;97(24):e11071. doi:10.1097/MD.0000000000011071
43. Cohen-Sacher B, Lerman-Sagie T, Lev D, Malinger G. Sonographic developmental milestones of the fetal cerebral cortex: a longitudinal study. Ultrasound Obstet Gynecol. 2006;27(5):494-502. doi:10.1002/uog.2757

44. Toi A, Lister WS, Fong KW. How early are fetal cerebral sulci visible at prenatal ultrasound and what is the normal pattern of early fetal sulcal development? Ultrasound Obstet Gynecol. 2004;24(7):706-15. doi:10.1002/uog.1802

45. Pistorius LR, Stoutenbeek P, Groenendaal F, de Vries L, Manten G, Mulder E, Visser G. Grade and symmetry of normal fetal cortical development: a longitudinal two- and three-dimensional ultrasound study. Ultrasound Obstet Gynecol. 2010;36(6):700-8. doi:10.1002/uog.7705

46. Malinger G, Kidron D, Schreiber L, Ben-Sira L, Hoffmann C, Lev D, et al. Prenatal diagnosis of malformations of cortical development by dedicated neurosonography. UItrasound Obstet Gynecol. 2007;29(2):178-91. doi:10.1002/ uog.3906

47. Malinger G, Lev D, Lerman-Sagie T. Abnormal sulcation as an early sign for migration disorders. Ultrasound Obstet Gynecol. 2004;24(7):704-5. doi:10.1002/uog. 1795

48. Chen X, Li SL, Luo GY, Norwitz ER, Ouyand S-Y, Wen H-X, et al. Ultrasonographic characteristics of cortical sulcus development in the human fetus between 18 and 41 weeks of gestation. Chin Med J (Engl). 2017;130(8):920-8. doi:10.4103/0366-6999.204114

49. Pooh RK, Machida M, Nakamura T, Uenishi K, Chiyo H, Itoh $\mathrm{K}$, et al. Increased Sylvian fissure angle as early sonographic sign of malformation of cortical development. Ultrasound Obstet Gynecol. 2019;54(2):199-206. doi:10.1002/uog.20171

50. Poon LC, Sahota DS, Chaemsaithong P, Nakamura T, Machida $\mathrm{M}$, et al. Transvaginal three-dimensional ultrasound assessment of Sylvian fissures at 18-30 weeks' gestation. Ultrasound Obstet Gynecol. 2019;54(2):190-8. doi:10.1002/ uog.20172

51. Lerman-Sagie T, Malinger G. Focus on the fetal Sylvian fissure. Ultrasound Obstet Gynecol. 2008;32(1):3-4. doi:10.1002/uog.5398

52. Guibaud L, Lacalm A. Etiological diagnostic tools to elucidate 'isolated' ventriculomegaly. Ultrasound Obstet Gynecol. 2015;46(1):1-11. doi:10.1002/uog.14687

53. Quarello E, Stirnemann J, Ville Y, Guibaud L. Assessment of fetal Sylvian fissure operculization between 22 and 32 weeks: a subjective approach. Ultrasound Obstet Gynecol. 2008;32(1):44-9. doi:10.1002/uog.5353

54. Guibaud L, Selleret L, Larroche JC, Buenerd A, Alias F, Gaucherand $\mathrm{P}$, et al. Abnormal Sylvian fissure on prenatal cerebral imaging: significance and correlation with neuropathological and postnatal data. Ultrasound Obstet Gynecol. 2008;32(1):50-60. doi:10.1002/uog.5357

55. Ben-Sira L, Garel C, Malinger G, Constantini S. Prenatal diagnosis of spinal dysraphism. Childs Nerv Syst. 2013;29(9):1541-52. doi:10.1007/s00381-013-2178-5

56. Malinger G, Lev D, Lerman-Sagie T. The fetal cerebellum. Pitfalls in diagnosis and management. Prenat Diagn. 2009;29(4):372-80. doi:10.1002/pd.2196 
57. Guibaud L, des Portes V. Plea for an anatomical approach to abnormalities of the posterior fossa in prenatal diagnosis. Ultrasound Obstet Gynecol. 2006;27(5):477-81. doi:10.1002/ uog. 2777

58. Lerman-Sagie T, Prayer D, Stöcklein S, Malinger G. Fetal cerebellar disorders. Handb Clin Neurol. 2018;155:3-23. doi:10.1016/B978-0-444-64189-2.00001-9

59. Leibovitz Z, Haratz KK, Malinger G, Shapiro I, Pressman C. Fetal posterior fossa dimensions: normal and anomalous development assessed in mid-sagittal cranial plane by three-dimensional multiplanar sonography. Ultrasound Obstet Gynecol. 2014;43(2):147-53. doi:10.1002/uog.12508

60. Leibovitz Z, Shkolnik C, Haratz KK, Malinger G, Shapiro I, Lerman-Sagie T. Assessment of fetal midbrain and hindbrain in mid-sagittal cranial plane by three-dimensional multiplanar sonography. Part 1: comparison of new and established nomograms. Ultrasound Obstet Gynecol. 2014;44(5):575-80. doi:10.1002/uog. 13308

61. Leibovitz Z, Shkolnik C, Haratz KK, Malinger G, Shapiro I, Lerman-Sagie T. Assessment of fetal midbrain and hindbrain in mid-sagittal cranial plane by three-dimensional multiplanar sonography. Part 2: application of nomograms to fetuses with posterior fossa malformations. Ultrasound Obstet Gynecol. 2014;44(5):581-7. doi:10.1002/uog.13312

62. Baumeister LA, Hertzberg BS, McNally PJ, Kliewer MA, Bowie JD. Fetal fourth ventricle: US appearance and frequency of depiction. Radiology. 1994;192(2):333-6. doi:10.1148/radiology.192.2.8029392

63. Quarello E, Molho M, Garel C, Couture A, Legac MP, Moutard $M L$,et al. Prenatal abnormal features of the fourth ventricle in Joubert syndrome and related disorders. Ultrasound Obstet Gynecol. 2014;43(2):227-32. doi:10.1002/uog.12567

64. Haratz KK, Shulevitz SL, Leibovitz Z, Lev D, Shalev J, Tomarkin $M$, et al. Fourth ventricle index: sonographic marker for severe fetal vermian dysgenesis/agenesis. Ultrasound Obstet Gynecol. 2019;53(3):390-5. doi:10.1002/uog.19034

65. Parisi MA. The molecular genetics of Joubert syndrome and related ciliopathies: The challenges of genetic and phenotypic heterogeneity. Transl Sci Rare Dis. 2019;4(1-2):25-49. doi:10.3233/TRD-19004

66. Poretti A, Boltshauser E. Fetal diagnosis of rhombencephalosynapsis. Neuropediatrics. 2015;46(6):357-8. doi:10.1055/s-0035-1566754

67. Desai S, Desai T. Prenatal diagnosis of pontocerebellar hypoplasia associated with rare syndromes: Expanding the genetic and phenotypic spectrum. Ultrasound Obstet Gynecol. 2021;57(3):498-9. doi: 10.1002/uog.22038.

68. Lacalm A, Nadaud B, Massoud M, Putoux A, Gaucherand P, Guibaud L. Prenatal diagnosis of cobblestone lissencephaly associated with Walker-Warburg syndrome based on a specific sonographic pattern. Ultrasound Obstet Gynecol. 2016;47(1):117-22. doi:10.1002/uog.15735

69. Lerman-Sagie T, Leibovitz Z. Malformations of cortical development: From postnatal to fetal imaging. Can J Neurol Sci. 2016;43(5):611-8. doi:10.1017/cjn.2016.27

70. Pertl B, Eder S, Stern C, Verheyen S. The fetal posterior fossa on prenatal ultrasound imaging: normal longitudinal development and posterior fossa anomalies. Ultraschall Med. 2019;40(6):692-721. doi:10.1055/a-1015-0157 